\title{
Investigation and Modeling of Two Phase Flow through a Compressor Stage: Analysis of Film Breakup
}

\author{
Niklas Neupert ${ }^{1 *}$, Hassan Gomaa, Franz Joos ${ }^{1}$, Bernhard Weigand ${ }^{2}$
}

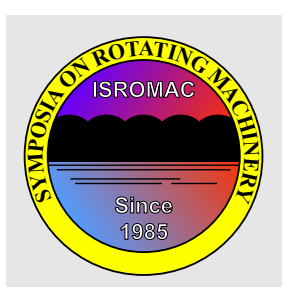

ISROMAC 2016

International

Symposium on Transport

Phenomena and

Dynamics of

Rotating Machinery

Hawaii, Honolulu

April 10-15, 2016

\begin{abstract}
For stationary gas turbines, the injection of water sprays into the compressor inlet is used to rapidly increase the power output. To fully utilize this technique a comprehensive understanding of the underlying phenomena is needed. An in-house CFD program was developed at Institute of Aerospace Thermodynamics (ITLR) Stuttgart which was validated on the transonic wind tunnel at Helmut Schmidt University (HSU) Hamburg. The present paper focuses on the water film on the blades formed by water deposition of impinged droplets. Experimental results using a reflective shadowgraphy method reveal the wall film pattern on the blade without disturbing the flow. A numerical model is presented predicting the position of film breakup based on linear stability analysis. The film breakup position is analyzed for three different water loads showing an almost constant position for the onset of film breakup in the experiments which is well captured by the numerical model.

\section{Keywords}

Two Phase Flow - Overspray Fogging — Experimental Investigation — Numerical Simulation

\author{
${ }^{1}$ Laboratory of Turbomachinery (LSM), Helmut-Schmidt-University, University of the Federal Armed Forces, Hamburg, Germany \\ ${ }^{2}$ Institute of Aerospace Thermodynamics, Stuttgart University, Stuttgart, Germany
}

*Corresponding author: Neupert@hsu-hh.de
\end{abstract}

\section{INTRODUCTION}

The increasing share of renewables in the electric energy producing sector increases the fluctuation of the power supply in the grid. To compensate this the requirements for conventional power plants move towards fast additional power and higher flexibilities [1]. The injection of a droplet spray into the inlet of the gas turbine, called inlet fogging, has shown to reduce compression work and increase mass flow by reducing gas turbine inlet temperature. This effect can be increased by injecting a higher fraction of water which is taken into the compressor and reducing the temperature rise throughout a compressor stage [2]. For instance this increase in flexibility helped to fulfill the stringent requirements of the UK grid code by providing an additional extra output of $18 \mathrm{MW}$ for the Marchwood power plant within a short period of time[3]. Small droplets in the range of $\leq 10 \mu \mathrm{m}$ are favorable due to a better follow-up behavior and higher ratio of surface to volume thus improved evaporation behavior. Despite the very low average diameter of the spray normally produced by overspray fogging systems (mean diameter $D_{10} \leq 10 \mu \mathrm{m}$ larger droplets in the range of $50-100 \mu \mathrm{m}$ can evolve from droplet droplet interaction and the entrainment of previously deposited water [4]. These larger droplets show a significantly larger characteristic time $\tau_{c}$ and thereby a longer duration to adapt a change in motion. This results in interactions with the structure leading to erosion problems and the formation of small secondary droplets caused by splashing. Depending on the impact conditions a significant amount of water is deposited on the blades leading to the formation of a wall film and streaks which are driven towards the trailing edge. The contribution of water on the blade and shape of the water air interphase has been shown to influence the overlaying air flow [5]. The presence of water increases the friction coefficient of the surface [6] suggesting a positive effect for smaller fraction of water covered surface, e.g. rivulets instead of a continuous film. In contrary the maximum height of rivulets is larger compared to the film due to continuity reasons. This would lead to the conclusion that depending on the boundary layer thickness of the air flow either a continuous film or discrete rivulets will have a stronger influence on the surrounding airflow [5]. In any case a prediction of the water flow pattern on the surface is detrimental for the comprehensive description of a two phase flow in a compressor. This aspect of a two phase flow problem in a compressor is addressed in this paper.

A direct measurement of the wall film properties in a gas turbine compressor under overspray fogging conditions is extremely difficult or even impossible. This necessitates the use of numerical tools for the investigation of the problem. To take into account small scale processes of single droplets within the numerical study of the whole compressor flow it is common to apply the Euler-Lagrange approach. While the flow field of the compressor cascade is computed by solving the Navier-Stokes equations, the behaviour of the droplets is computed by solving the equations of motion for parcels and the resulting trajectories. In the case that a droplet trajectory hits a blade a subscale model is necessary that describes the drop impact phenomenology. This model has to provide an 
elaborate prediction of e.g. the ejected secondary droplets' diameter. Applying this to a big amount of droplets yields the droplet diameter spectrum of the spray along the blade. Additionally, the model facilitates the quantification of the specified film properties.

In a previous study the outcome of the droplet wall interaction was presented for a flat plate [7]. This model determines the deposited water mass on the blade, the resulting film thickness and film velocity. In this study the model is extended to account for the position of film breakup based on the damping of small perturbations imposed on the water film.

Due to the high complexity of a two-phase compressor flow these tools are restricted to the assumptions the respective model was derived for. Therefore a comparison to experimental data derived in a simplified and therefore measurable environment is still inevitable. This simplified environment was chosen to be a linear compressor cascade under overspray fogging conditions. The position of film breakup was determined by quantitative optical evaluation of the suction side wall film on the compressor blades. For the evaluation of the flow pattern an image processing algorithm was developed. In section 1 the experimental setup is explained including the description of the measurement system. The next paragraph is dedicated to explain the image processing algorithm. The algorithm was validated with a test target with different backgrounds to give an insight in the accuracy of the used method. Afterwards the numerical model is explained consisting of the description of the multiphase code, the wall film and the perturbation model. Finally, a test case is chosen to compare the experimental and numerical evaluation of the breakup position.

\section{EXPERIMENTAL SETUP}

The experiments were conducted at the open loop transonic wind tunnel at the Laboratory of Turbomachinery at the Helmut-Schmidt University in Hamburg, Germany, as sketched in fig. 1 and described in detail in a previous publication [8]. For clarification only a short summary of the most important features shall be given here. The air is supplied by two radial compressors and cooled by a heat exchanger to maintain a constant inlet total temperature in the settling chamber of $T_{\text {tot }, \text { in }}=304 \mathrm{~K}$. Deionized water is injected in the settling chamber via a pin jet nozzle array (BETE PJ 10 nozzles) with up to 30 nozzles. The spectrum of a spray is characterized by a representative diameter and a diameter describing the range of droplet sizes [9]. The spray produced by the pin jet nozzles has a mean diameter $D_{10}=7.0 \mu \mathrm{m}$ and a Sauter Mean Diameter $D_{32}=38.7 \mu \mathrm{m}$ at the inlet of the cascade. The diameters are calculated with the following formula:

$$
D_{a b}=\frac{\Sigma N_{i} D_{i}^{a}}{\Sigma N_{i} D_{i}^{b}}
$$

Regarding the small $D_{10}$ the majority of the droplets is very small which is in accordance with the manufacturer and industry standards for overspray fogging systems in use as shown by Chaker and Meher-Homji [10]. However, the mass averaged

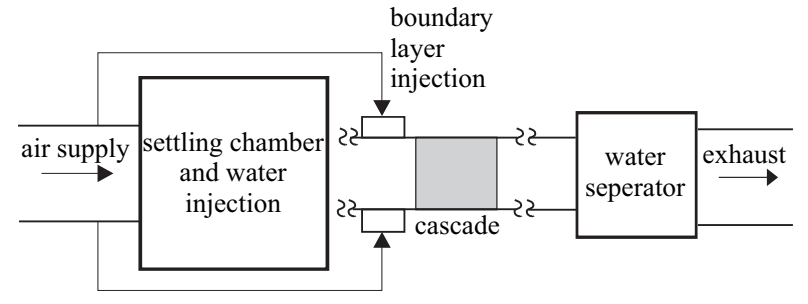

Figure 1. Sketch of the test rig (top view)

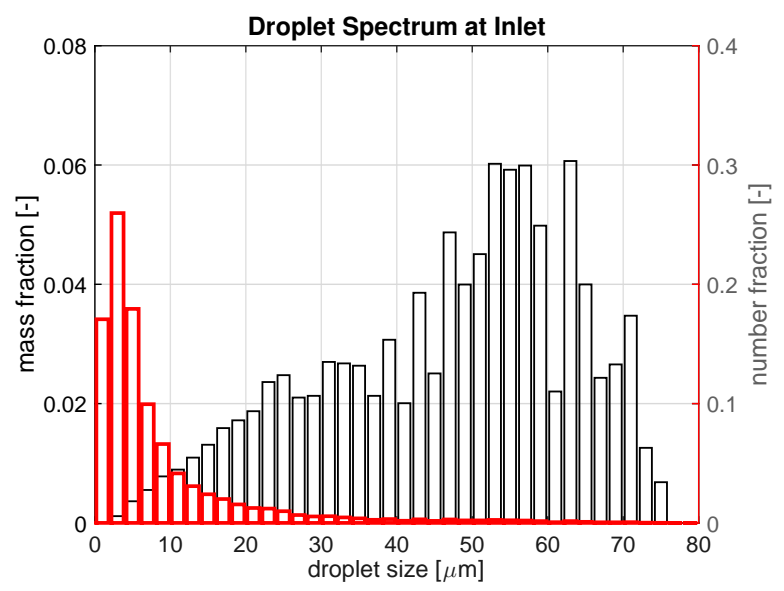

Figure 2. Inlet Droplet Spectrum

distribution shown in fig. 2 reveals the issue of having a very low number of larger droplets in the flow which contain a high fraction of total water mass. Due to their relatively high inertia exactly these droplets will not follow the gas flow closely, hit the blade and contribute significantly to the deposited water mass on the blade and a higher risk of erosion. This emphasizes the need to report two values to characterize a spray ay stated in [9].

The two-phase flow is accelerated via a bell mouth into the inlet duct which has a length of approximately $2 \mathrm{~m}$. Due to this relatively long acceleration length the slip velocity between the droplets and the air flow becomes negligible. The drawback of this large extension of the inlet duct is a significant thickening of the sidewall boundary layer which has to be treated properly to maintain a quasi-2D flow in the test section. Therefore the test rig is equipped with a slit nozzle at the sidewall boundaries via which compressed air is injected in front of the cascade. The cascade consisting of eight CDA-profiles (Controlled Diffusion Airfoil) is mounted between two acrylic glass windows allowing optical accessibility to the test section. The geometric properties and design flow conditions are presented in Tab. 1. Additional optical access is provided by a quartz-glass window on top of the test section to provide a direct optical access to the suction side of the middle blade of the cascade. To extract the water from the flow a separator is installed approximately $7 m$ downstream of the cascade. 
Table 1. Cascade properties

\begin{tabular}{lc}
\hline Property & Outer Cross Section \\
\hline Chord & $50 \mathrm{~mm}$ \\
Pitch & $50 \mathrm{~mm}$ \\
$\Delta \beta_{\text {design }}$ & $6.0^{\circ}$ \\
$M a_{\text {design }}$ & 0.89 \\
$\left(w_{2} / w_{1}\right)_{\text {design }}$ & 0.75 \\
No. of blades & 8 \\
\hline
\end{tabular}

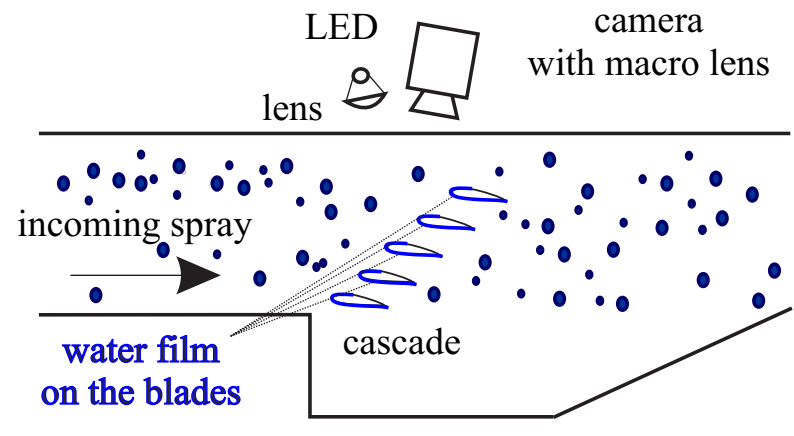

Figure 3. Measurement setup

To ensure constant boundary conditions during the tests several static and total pressure and temperature probes as well as humidity probes are mounted in the test rig. Furthermore, static pressure is measured at several pitches in front of and behind the traverse to ensure a periodic flow in the region of interest. For the velocity and droplet diameter measurements a two dimensional Laser Doppler and Phase Doppler Anemometer (LDA/PDA) is used. By this technique the velocity components in two dimensions and the droplet diameter can be measured simultaneously with a maximum error of less than $3 \%$. More details of this technique can be found in [11].

The setup for the measurement of the wall film pattern is schematically shown in fig. 3. For the investigation of the wall film pattern a DSLR Camera (Canon 5D Mark III 22.3-Megapixel full frame-CMOS sensor (5760 x 3840 pixel at a $36 \mathrm{~mm} \times 24 \mathrm{~mm}$ sensor)) with MACRO-lens (Canon EF $180 \mathrm{~mm} \mathrm{f/3.5L}$ Macro with $1: 1$ magnification factor, depth of field $\approx 0.02 \mathrm{~mm}$ ) was used. Thereby it was possible to derive images of the wall film flow pattern in a high resolution of $6.25 \mu \mathrm{m} /$ pixel without any disturbance of the flow field in the cascade due to additional obstacles. The camera is mounted on a support which is adjustable in all three dimensions and rotatable around all three axis to perfectly adjust the camera. Due to the small field depth of the lens translational stages with a high setting sensitivity are used (OWIS LT80). Due to the fact that the film structure is almost stationary the minimum shutter time of the camera is sufficiently short to capture a picture without movement blur $\left(t_{\text {shutter }}=125 \mu \mathrm{s}\right)$. Therefore a constant source of light is chosen (white light LED Cree XR-E) which reduces the noise due to lower values of

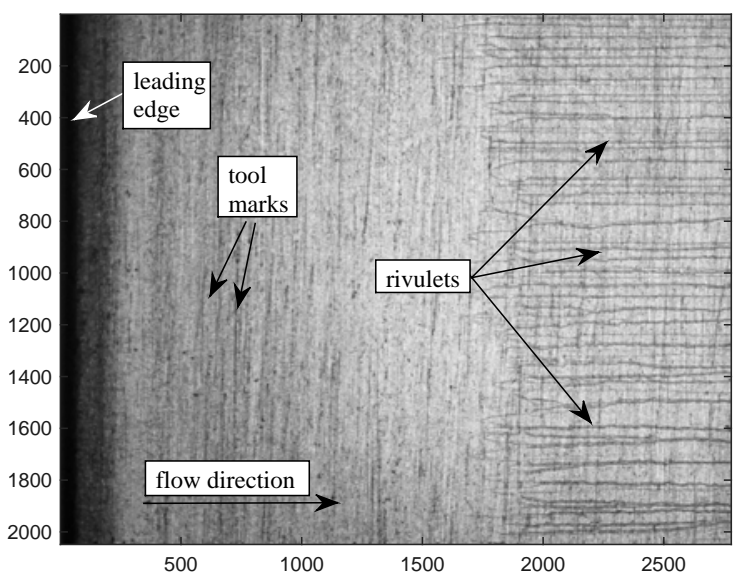

Figure 4. Gray Scale Image of Film Pattern

sensitivity of the sensor. The LED is fixed on the mount of the camera to maintain a good alignment of the optical axis of the LED, the blade surface and the camera. To reduce the error due to unwanted light sources the test section is completely darkened during the recording of the images. The uncertainty of the setup is strongly dependent on the experimental environment. Therefore the setup was tested under different boundary conditions to estimate the measurement uncertainty. The results of this are presented in section 1.2. The details of the post-processing is presented in the next section.

\subsection{Diagnostics}

An image processing code was written using MATLAB to evaluate the water structures on the blade. Figure 4 shows the unprocessed image. The incoming light of the diode is reflected from the surface of the blade. If water is present the light is refracted at the gas liquid interface. In the case of small rivulets this interface is curved and the light coming from the top is scattered sidewards. Thereby less light reaches the sensor and these streaks appear dark. The point of film breakup is therefore defined as the starting positions of the water rivulets. In general this task is quite similar to general object detection tasks in industrial image processing or in PIV image analysis.

The process in the code can be divided into four major steps. The first step contains the pre-processing of the image with different filter methods resulting in a gray scale image with improved contrast and reduced background noise. In the second step objects are detected leading to a binary matrix of the image. In the third step a quasi morphological filter is used to reduce noise. The fourth step is the measurement of the film length. This is done by evaluating the binary matrix in terms of size of objects and the distances between them. These steps shall be briefly explained in the following paragraphs.

First the image is transferred to a gray scale image which is a matrix of brightness values of $0-255$. The task is 


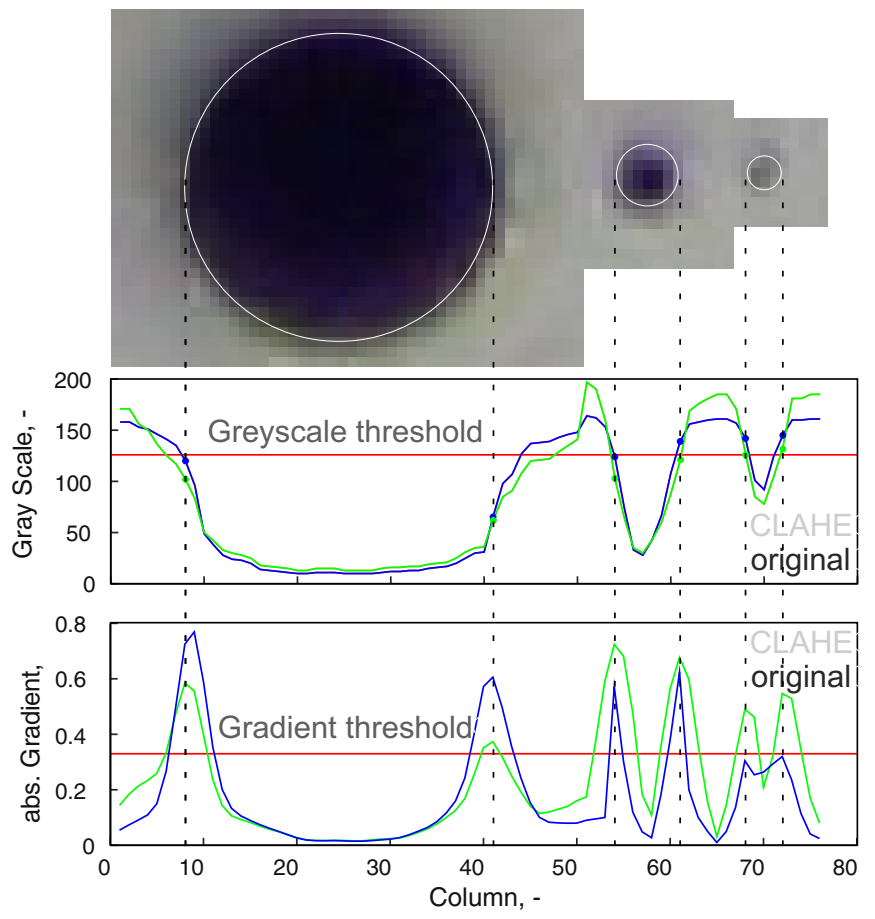

Figure 5. Details of the detection algorithm

to find the dark areas in the image. Afterwards the gray scale image is corrected with a reference image taken in the same setup without the presence of water to distinguish between background noise and the wanted objects. Usually a subtraction of the images can be done. The resulting matrix will have negative values in regions where light was scattered and lesser light reaches the sensor and accordingly positive values for the brighter regions. The background is blurred out. If the background has a highly nonuniform distribution of brightness the matching of the measuring image and the background image can be challenging especially when the medium in the area between reflective surface and lens is changed due to the presence of water. The water changes the exact position and size of the background noise such as tool marks and leads to an inconsistent reduction of noise. Further very small structures with a low level of scattered light are blurred out. Therefore an alternative background correction was applied. The background image was filtered for dark regions (tool marks which scatter the light) that are blurred with a Gaussian filter. This image is finally subtracted from the image of interest. Thereby a significant increase of the detectability of the rivulets could be achieved.

To further increase the detectability of small structures and to ensure a global threshold the contrast of the image was adapted using a Contrast Limited Adaptive Histogram Equalization (CLAHE) Method which is already implemented in the Matlab Image Processing toolbox [12]. The method locally applies a mask with a given size and linearly adapts the brightness of the picture to the maximum range of the histogram. Figure 6 shows the effect of the CLAHE filter on the gray scale level and the gradients in the image. Depending on the size of the applied mask the gradients and the difference of the gray scale of background and object is increased for smaller objects. The drawback is an increase of background noise because every imbalance is enhanced. Challenging in the analysis of water structures on the blade is the presence of tool marks on the blade which cannot be totally extinguished with the background correction method. However, these run mostly in parallel direction to the leading edge whereas the rivulets run almost perpendicular to these marks. This helps to distinguish between rivulets and tool marks. For the reduction of these signals the image was averaged in perpendicular direction to the tool marks. A block-wise averaging was conducted. Following this averaging the image a modified Canny edge detection is applied [13]. This algorithm returns a binary image in which the edges are marked with ones. Basic steps of this method are smoothing the image with a Gaussian filter, calculating the gradients in the picture in both directions with a Sobel filter, finding the local maximum of the detected zone and thinning the detected edge to a singular pixel. For the detection of the rivulets the Canny filter was changed to only consider the gradients in direction of the leading edge. Thereby the tool marks running perpendicular direction are not detected. In a further step the magnitude of the gradient is averaged over a connected edge and a filter is applied to delete edges with a low value. This allows a relatively low threshold in the Gaussian filter while reducing the noise of too many detected structures. In a last step in the filtering algorithm the detected edge itself is checked based on the gray scale value to check if the position of the detected edge belongs to the object.

A drawback of the Canny edge detection is the fact, that junctions of edges are not detected. A closed line of breakup cannot be detected so far. To distinguish between the area on the blade which is covered by a continuous film or by rivulets the found edges are thickened which leads to the connection of the edges in the region in which multiple edges are detected. A consequent erosion of the binary image for large structures leads to the deletion of unwanted noise. In a following step the edge detection is repeated in the roughly determined area of breakup with a lower threshold. This leads to an increased accuracy in the determination of the breakup line. The leading edge is detected using several criteria. First of all the images are rotated in the same position resulting in a position of the leading edge at the right end of the image. Furthermore, the leading edge appears as a very dark area caused by the high curvature and the resulting reflection of the light away from the sensor. Finally the leading edge is an almost perfectly straight line which directly leads to the application of a morphological filter. In this code a Hough-transformation is used to detect the leading edge.

As can be seen the method relies on thresholds which are crucial for the accuracy. Therefore the program was applied for two different test cases in which the size of the objects is known a priori. Thereby a quantitative evaluation of the results is more reliable. 

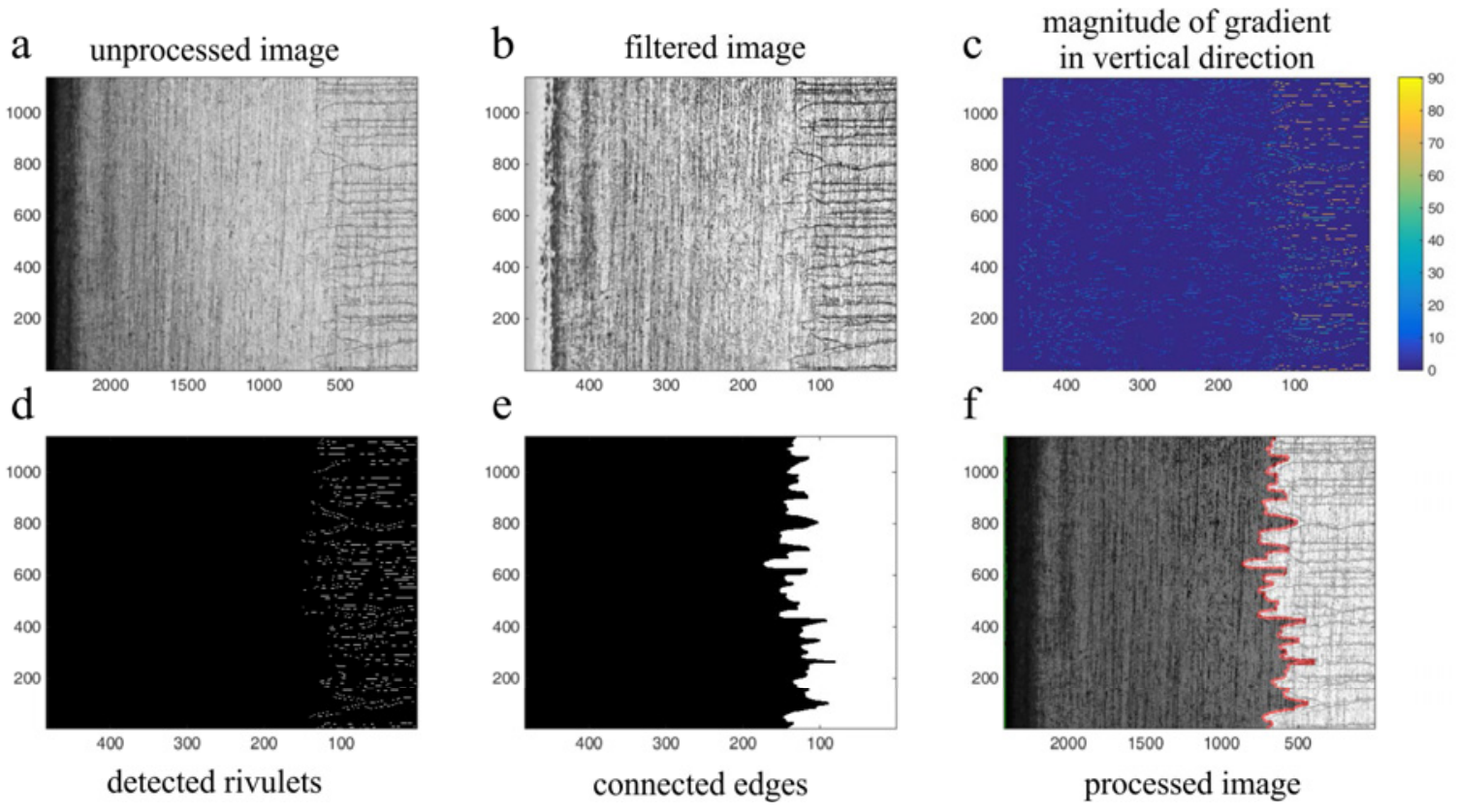

Figure 6. Stepwise detection of film breakup position. (a) original image, (b) after adaption of background correction, CLAHE filter and averaging over 5 pixels in horizontal direction, (c) detected edges with modified Canny edge detection method colored with the magnitude of the gradient in vertical direction, (d) binary image of detected rivulet edges, (e) binary image of connected edges yielding in rivulet area, (f) final image with detected leading edge (green) and breakup position (red)

\subsection{Calibration Targets}

The test object was a calibration glass plate with opaque circles normally used for depth of field calibration for shadowgraphy systems (LaVision calibration target for ParticleMaster Calibration). The plate is divided into 4 regions each region containing circles in different size classes ranging from $10-200 \mu \mathrm{m}$ for region 1 to $100-2000 \mu \mathrm{m}$ for region 4 . The dimensional accuracy of the circles is in sub-micron region. It has to be mentioned that the code had to be adapted slightly. Due to the fact that the only calibration target having a sufficiently high accuracy available so far displays discs instead of lines the quasi morphological aspects of the codes were blanked out (e.g. neglecting the gradient in the Canny filter in $x$-direction and the averaging of the structures in the $x$-direction). Nevertheless, these steps do not address the accuracy but only the noise reduction of the code. Therefore a calibration having different structures than the final image is still valid for the determination of the accuracy.

The plate was positioned on a nearly uniform white background (test case 1) and on the blade in the final test setup without any air flow present (test case 2). The cut-outs of the test images are shown in fig. $7 \mathrm{a}$ and c. Due to the curved surface of the blade the shadow of the discs increase the size of the detected objects to an unknown value and led to an additional error. Therefore the area of interest (AoI) had to be limited to the center region in the second case. Until now no flexible object with an adequate accuracy was present to give more accurate results. Future tests will include a flexible test object which counteracts this effect.
As mentioned in the last paragraph the image process algorithm depends on some thresholds. A thorough variation over a reasonable range of those filters was applied to determine the most fitting input parameters for the program. A variation of the input parameters showed a minor dependency of the results towards the size of the CLAHE mask in a reasonable range (max $24 \times 24$ pixels). As seen in fig. 6 the threshold of the gradient filter in the Canny algorithm had to be adapted to the impact of the CLAHE filter. A further variation, however, showed vary stable results for a fixed value of 0.35 for the gradient filter. This seems to be reasonable due to the fact that CLAHE filter changes the range of the histogram to the maximum dimension (256 bins) and that the edges in the test target are designed to be very sharp so that the gradients are quite high. Therefore, only the influence of the size of the Gaussian filter in the Canny edge detection is discussed in detail to show the accuracy of the algorithm under different background conditions.

Figure 8 shows the results for both test cases. The first evaluation criterion for the tests are chosen to be the relative deviation of the measured size and the range of the measurement in each class, shown in fig. 8 a and d. The second is the percentage of detected objects (number of detect objects in each class divided by the number of objects on calibration target) for the determination of the range of the measurement ability and the completeness in each size region and as a third parameter the percentage of falsely detected objects to show the robustness of the parameter setting.

The accuracy of the method is shown in fig. 8 a and d. The 

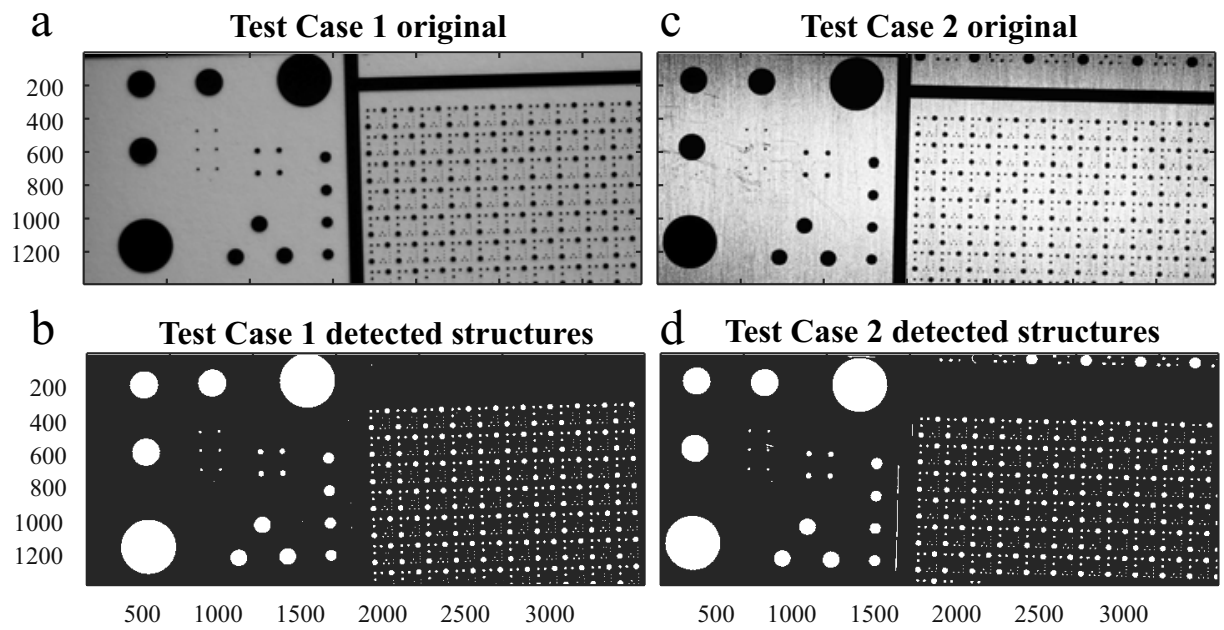

Figure 7. Calibration target with white background (a-b) and blade surface (c-d). a and c displays cutouts of the input images, b and $d$ the detected objects.

black line corresponds to an error of \pm 2 pixel. As can be seen in both graphs in the first row of fig 8 the accuracy of the detected particles lies within \pm 2 pixels showing reasonably small deviations over the whole range of detected particles. Further the size of the objects is slightly overestimated for large particles and underestimated for small particles. This is caused by the adaption of a constant gray scale threshold as seen in fig. 6 and also reported in [14] for a similar problem in the image processing of spray analysis. In [14] the issue was approached with an adaptive threshold for a given particle size. Due to the fact that the tool is meant to detect rivulets which are long and thin streaks instead of round shapes this influence on the accuracy of the measurement could not be estimated at this point. Contrary to the case of a small droplet the scattering of light is stronger in case of the rivulets because light reflected from the surface comes only from one direction, e.g. top and bottom, whereas in case of the small round objects the light comes from all sides. Therefore no size adaption of the threshold was implemented. The application of a test target with lines will help solving this problem. The increase of the mean diameter in case of the smallest detected particle for a given filter size results from the fact that objects which are detected to be in the lower range of the object class are not detected anymore which shifts the averaged diameter to larger values. Due to the low background noise in test case 1 even small discs in the range of $20 \mu \mathrm{m}$ can be measured with an accuracy $\pm 20 \% \equiv 0.6$ pixel. For the second test case the optimal setting in the detection has been chosen and the size of the Gaussian filter has been varied. The result for the same parameters filter size of $\sigma=1.25$ showed a high level of noise. As it is expected a larger smoothing filter leads to a significant reduction of noise and to a higher accuracy in the measured diameter also for larger droplet classes. The drawback is that no more elements in the $20 \mu \mathrm{m}$ class could be detected.

Figure $8 \mathrm{~b}$ and e show the percentage of detected particles for both test cases. It shall be noted that the range of the abscissa is reduced to a maximum of $100 \mu \mathrm{m}$. For the test case 1 the fraction of detected particles in the $20 \mu \mathrm{m}$ class is larger than 0.95 and reaching 1 for the $40 \mu \mathrm{m}$ discs. For larger filters value of detected particles reduces to 0.78 and 0 for the largest Gaussian filter tested. For particle classes larger than $60 \mu \mathrm{m}$ every disc is detected. With increased background noise in case of the target positioned on the blade the detectability is significantly reduced. This is caused by a larger gradient threshold of 0.35 (compared to 0.1 for white background) which had to be applied to reduce the background noise. The number of detected objects in case of the $20 \mu \mathrm{m}$ class is negligibly small so that the first validated signal is in the $40 \mu \mathrm{m}$ class. For the $60 \mu \mathrm{m}$ class, however, every disc was detected with the smallest and medium filter.

The percentage of erroneously detected particles is shown fig. $8 \mathrm{c}$ and $\mathrm{f}$. For both cases the level of noise is negligibly small (4.7\% erroneously detected objects for a white background and $2.1 \%$ for the blade surface). The adaption of larger smoothing filters further reduces this value to a negligible level.

This section has shown that the setup is capable of measuring small objects (down to $20 \mu \mathrm{m}$ ) with a reasonable accuracy $( \pm 20 \%)$. For the test setup the increase of background noise increases the lower limit of detectability to $40 \mu \mathrm{m}$ (with an accuracy of $\pm 10 \%$ ). Due to the arguments mentioned above that the blur in case of rivulets with a certain width is smaller compared to discs with the same diameter the accuracy seems to be sufficiently high for the determination of the position of film breakup.

\section{NUMERICAL MODELING}

The numerical work flow applied here to capture the liquid dynamics is to a large extent particularly tailored for the present application of droplet laden compressor flows. In the following the focus lies on the treatment of the wall film formation and propagation along the blade and only a brief overview can be given for remaining modeling. A detailed treatment of 

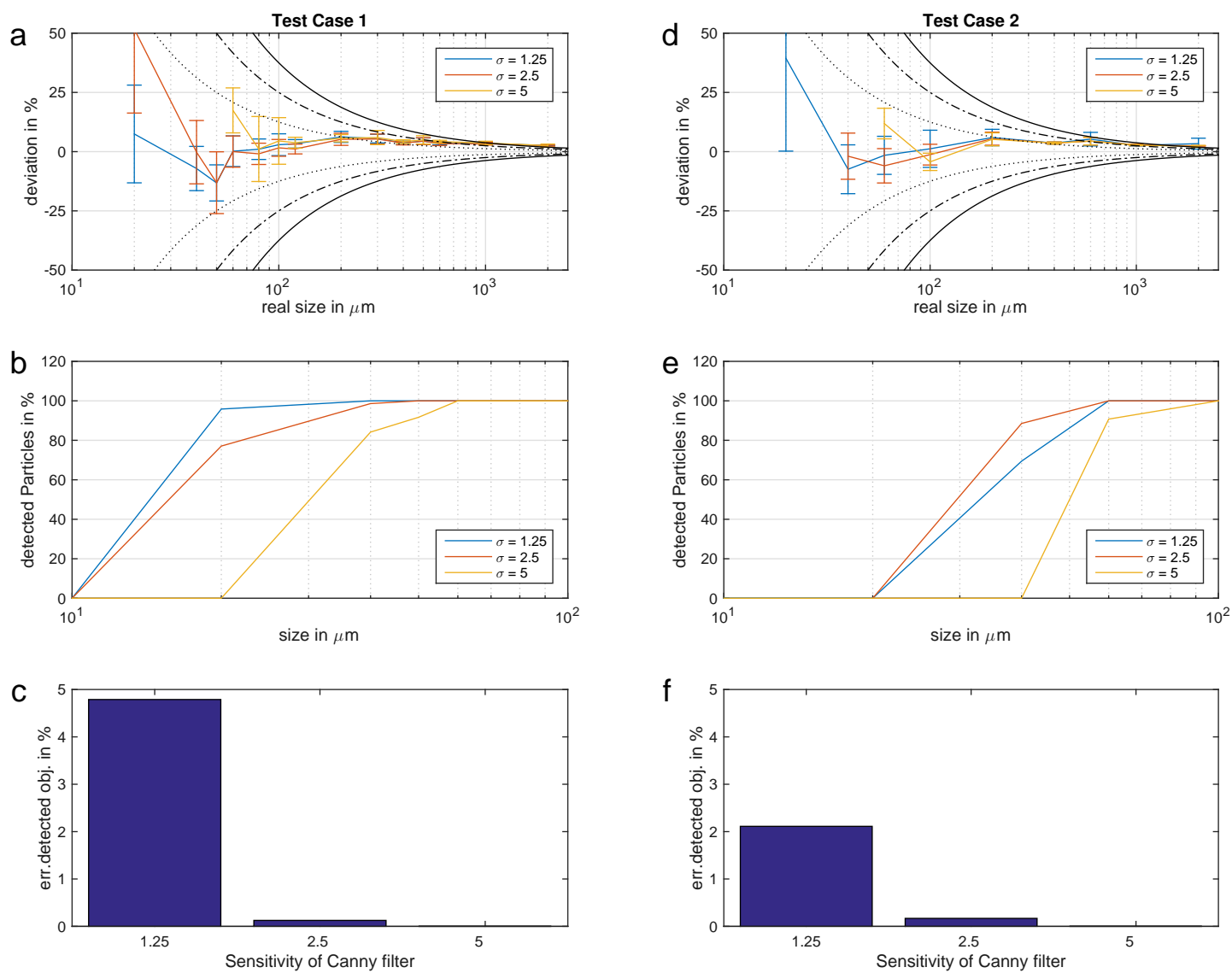

Figure 8. Variation of Gaussian filter in the Canny edge detection algorithm. Results for white background (test case 1, a-c) and blade surface (test case 2, d-f). The mean deviation with error bars showing the range of $2 \sigma$ are displayed in a and d the enveloping curves represent $\pm 2,4,6$ pixels. $\mathrm{b}$ and e display the percentage of detected objects over the diameter. $\mathrm{c}$ and $\mathrm{f}$ show the percentage of additional detected structures (noise)

the entire work flow and the derivation of the sub-models is given by Gomaa [15]. To capture the spray motion through the carrier gas flow domain the Euler-Lagrange approach is applied. At first the dry gas flow solution is computed with ANSYS CFX 14.0 [16] solving RANS equations to establish the carrier gas velocity field as well as the gas flow related boundary conditions for the wall film flow. The droplet trajectories result from 4th order numerical integration [17] of the Lagrangian equations of motion, while simultaneously accounting for the Reynolds number dependency of the drag as well as the oscillating shape of the drops. Deformation and drop breakup are modeled in terms of the TAB model (Taylor Analogy Breakup) [18]. In the present work so called one way coupling is applied, thus the coupling of the continuous flow to the drop motion as well as interactions between droplets are neglected. To reduce computational effort droplets of equal properties are gathered in parcels [19]. All trajectories that hit the substrate contribute to the formation and spreading of the wall film as well as possible formation of secondary droplets that are ejected back into the flow. The splashing model comprises a threshold to predict whether secondary droplets are ejected back to the gas flow or not. In case that secondary droplets are formed, properties are provided in form of correlations for diameter and ejection speed distributions [15]. The remaining mass and momentum of the impacting spray contribute to the formation and propagation of a wall film along the blade. It can be shown that the present application typically involves film heights that are much smaller than characteristic scales in flow direction [15].To resolve the wall film behavior it is therefore sufficient to solve the conservation equations for mass and momentum in height averaged form. Furthermore, it was shown that wall film inertia is typically small and may usually be neglected. In that creeping flow regime the momentum equation can be solved analytically in closed from yielding the a single evolution equation for the 
wall film height

$$
\partial_{t} h+\nabla \cdot[\overline{\mathbf{u}} h]=\dot{h}_{I},
$$

where $h$ denotes the wall film height, $\overline{\mathbf{u}}$ the height average film velocity and $\dot{h}_{I}$ the net mass source term due to impacting droplets. The average film velocity results from the momentum equations to

$$
\overline{\mathbf{u}}=\frac{1}{\mu_{L}}\left(\frac{1}{2} h\left(\tau_{G}+\tau_{I}\right)-\frac{1}{3} h^{2} \nabla\left[p_{G}-\sigma\left(\kappa+\nabla^{2} h\right)-\phi\right]\right)
$$

Here $\mu_{L}$ denotes the liquid viscosity, $\tau_{G}$ and $\tau_{I}$ denote the shear stresses induced by the co-flowing gas and the impacting spray, respectively. Furthermore, the solution is affected by the gradient of the gas pressure $p_{G}$ and the potential $\phi$. The surface tension $\sigma$ affects the film at the occurrence of substrate curvature $\kappa$. Even though available in the present implementation [15], contact line modeling is not accounted for here for reasons to be discussed in section 3.2. The wall film evolution is solved on a substrate fixed curvilinear grid with standard finite volume up winding [15]. As already mentioned the number of computed trajectories is drastically reduced by applying parcels rather than droplets. In consequence impact induced mass and momentum source distributions become vastly discontinuous. Since they serve as boundary conditions for the film solution it is beneficial for the commonly continuous wall film solution to include a mass and momentum conservative smoothing algorithm to the according source terms [15].

Driven by gas shear stresses and impacting spray momentum the wall film propagates towards the trailing edge of the blade where the film disintegrates back into the flow. Based on different breakup modes the developed model [15] prescribes the size distribution of the ejected spray, where the continuous liquid film phase is transformed into discrete Lagrangian particles again.

\section{RESULTS AND DISCUSSION}

In this section the experimental and numerical results for a representative test case are presented. The design conditions of the cascade were chosen. The inlet flow Mach number was fixed to $M a_{i n}=0.89$ and the inlet flow angle to $\beta_{i n}=151^{\circ}$. The water load was changed between $\xi_{w}=0.5 \%, 1 \%$ and $1.5 \%$.

\subsection{Experimental Results}

For the evaluation of the position of film breakup five images for each water load were analyzed. The evaluation area was set to the center of the channel width and the size was maximized to the area of illumination. This results in a frame size of maximum $24 \mathrm{~mm}$ in stream wise direction and $25 \mathrm{~mm}$ in direction of the channel width. The size is sufficiently large to give reproducible and representative results for each case tested. As mentioned previously the position of film breakup

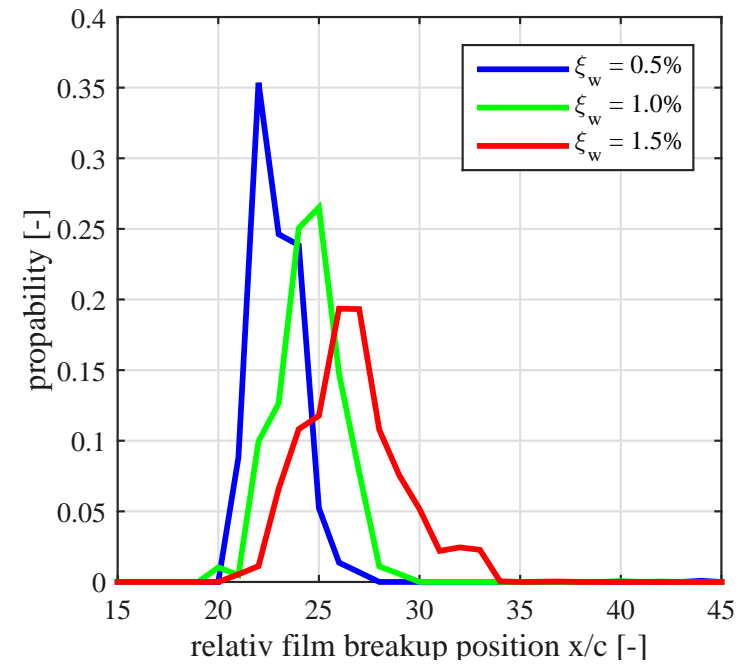

Figure 9. Experimental results for film breakup position (average of 5 images)

is defined as the position of a beginning rivulet with a width of the structure of less than $150 \mu m$.

Figure 9 shows a comparison of the normalized position of film breakup for different water loads. The curves represent the average over 5 images, respectively. It is seen that the onset of breakup is nearly independent of the water load. For each case the film ruptures at $x / c \approx 20 \%$. Contrary to the same position the highest possibility of breakup is shifted backwards slightly from $x / c=22 \%$ to $x / c=27 \%$ for an increasing water load. Further the position in which the transition to a rivulet flow pattern is completed is increased with a higher water load. Figures 10 (a)- (c) show representative examples of the analyzed images for a water load of $x i_{w}=0.5 \%, x i_{w}=1.0 \%$ and $x i_{w}=1.5 \%$. For the low water load the variation in the breakup position is very small leading to a maximum deviation in the position of $\Delta x / c= \pm 3 \%$ which corresponds to a variation of $\pm 1.5 \mathrm{~mm}$ in absolute coordinates. For higher water loads the position of film breakup is stretched to wider area. As can be seen in fig. 10 (b) the first occurrence of film breakup is located at approximately the same position. In between these early breakup positions, however, the water stays in a film flow pattern (width $>150 \mu \mathrm{m}$ ). These additional interfaces at the sides of the wider film streaks seem to stabilize the structures resulting in a delayed breakup. The result of this delayed breakup can be seen quantitatively in fig. 9. The area of breakup is increased to almost $\Delta x / c= \pm 7 \% \equiv \pm 3.5 \mathrm{~mm}$ for a water load of $\xi_{w}=1.5 \%$. Before analyzing these differences in more detail the numerical results shall give valuable insights on this issue.

\subsection{Numerical Results}

The numerical simulation is set up according to the experimentally investigated configuration at different water loadings of $\xi_{w}=0.5 \%, 1 \%, 1.5 \%$. The dry flow field is computed 


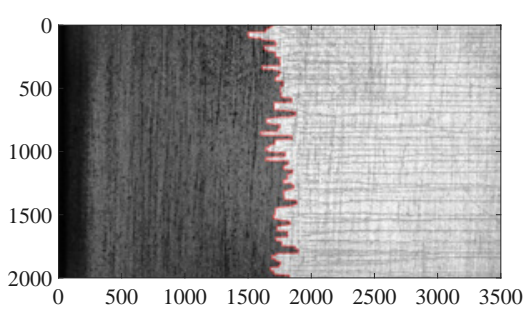

(a)

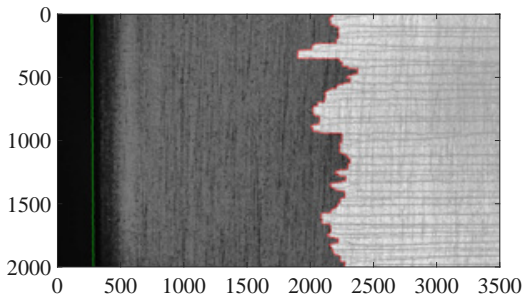

(b)

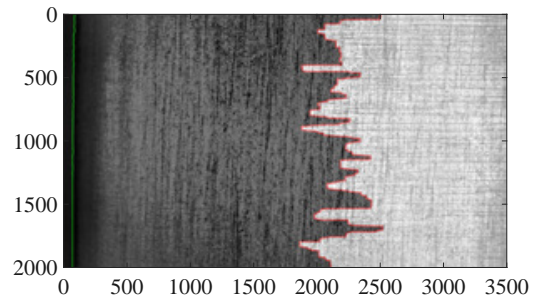

(c)

Figure 10. Processed image of water film patter on the blade suction side for a water load of $\xi_{w}=0.5 \%$ (a), $\xi_{w}=1.0 \%$ (b) and $\xi_{w}=1.5 \%$ (c). The white area detects the region of rivulets, the green line marks the blade leading edge and the red line represents the detected position of film breakup

with the commercial code ANSYS CFX and is simulated in a quasi $2 \mathrm{D}$ domain that covers the five central blades of the experimental rig, described in section 1 . The entire fluid domain is discretized by 46,860 cells, with respectively 5 cells in the lateral direction, that is bounded by symmetry conditions. Turbulence is modeled with the $k-\epsilon$ turbulence model using a scalable wall function. The injected drop spectrum corresponds to the experimentally determined diameter spectrum, shown in fig. 2, discretized in $1 \mu \mathrm{m}$ steps. Overall about 58,000 parcels are injected along the inlet plane without slip to the ambient gas and every trajectory is followed until it hits one of the blades or reaches the domain boundaries. The hitting trajectories determine the mass and momentum source terms along the blade as shown in fig. $11 \mathrm{a}$ ). The boundary conditions further include the gas induced shear stress. In the regarded setup the gas pressure gradient is only of minor influence. It shows a slight effect at the blade leading edge and is therefore not explicitly shown here, but yet incorporated in the solution. The variation of the water loading scales the induced liquid mass and momentum sources.

In fig. 12 the obtained solution for the wall film thickness and velocity is presented for different water loadings. The increased source terms at higher water loading translate into increased thicknesses and velocity of the wall film.

The development of lateral patterns, such as the formation of streaks is artificially suppressed here as the regarded setup is quasi two dimensional. To still obtain an indication on the breakup of the film for the present setup a linear stability analysis is applied to the wall film evolution equation. For that purpose the long scale solution of the wall film height $h_{0}$ that suffices the film evolution eqn. 2 is superimposed with a small disturbance $h^{\prime}$, thus $h=h_{0}+h^{\prime}$. It can be shown that after linearization the perturbation velocity generally relates to the mean film velocity by $u_{h}^{\prime}=\left.\partial_{h}[\bar{u} h]\right|_{h_{0}}$. In general a dependency between wave number and phase speed on the growth rate exists only if the particular term depends on the film thickness. As in the present formulation shear stresses, gas pressure and the substrate curvature are initially assumed to be independent of the film thickness; they equally affect the entire wave spectrum and no wave number dependency exists. The growth rate induced by these particular terms is (a)

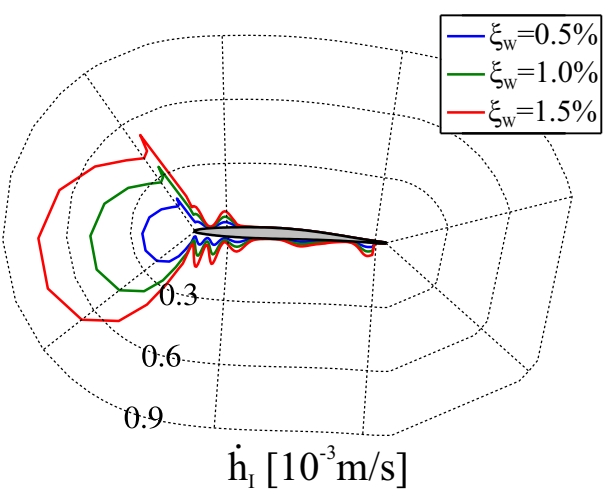

(b)

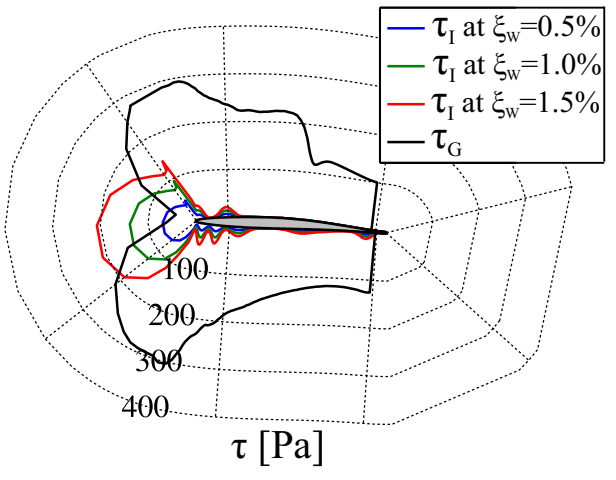

Figure 11. Boundary conditions for the wall film model in terms of mass and momentum source terms $\dot{h}_{I}$ (a) and wall shear $\tau_{G}$ and $\tau_{I}$ (b) 


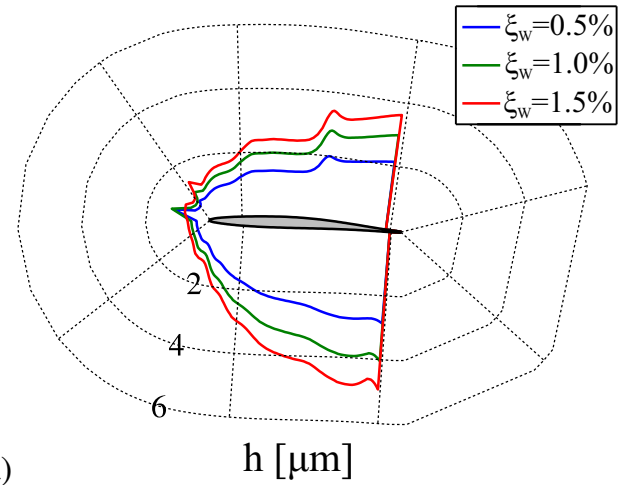

(a)

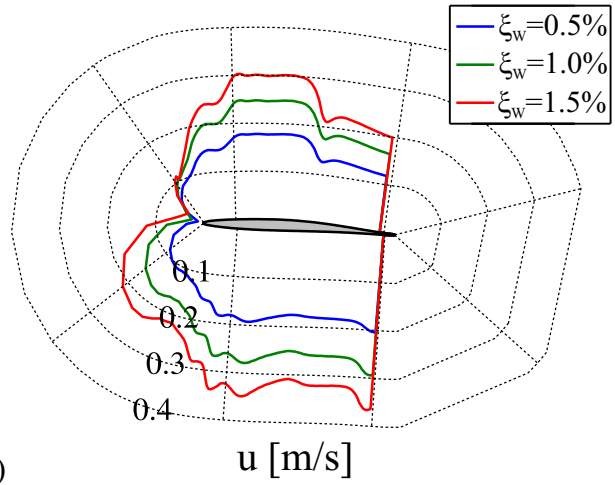

Figure 12. Wall film thickness $h$ (a) and wall film velocity $u$ (b)

equivalent to the slope of $u_{h}^{\prime}$, that is immediately determined for a computed solution $h_{0}$. The analysis of the given evolution equation shows that any exerted force that accelerates the wall film, corresponds to a negative growth rate, thus tends to stabilize the film, while a decelerating force incorporates a destabilizing effect. However, the analysis does not provide a definite criteria to predict the breakup, but rather allows an estimate where breakup is likely to occur.

In fig. 13 the perturbation velocity is shown and one may estimate that due to the deceleration, breakup seems probable on the suction side of the blade. Since, mostly influenced by the gas shear stress in the present setup, it is quite interesting to find that the deceleration and therefore the breakup position, does not seem to be affected by the different water loads.

\subsection{Discussion}

The numerical results show a strong reduction of the velocity $u$ and the perturbation velocity $u^{\prime}$ at $x / c \approx 60 \%$ on the suction side. This strong deceleration, however, corresponds to the shock-position and the consequent reduction of wall shear $\tau_{G}$. Due to the fact that the analysis is based on a $2 \mathrm{D}$ approach the film height $h$ increases. Following the above mentioned arguments a continuous film would breakup at the latest at this position. This strong influence of the shock on the wall film shall not be discussed further herein but will be scope of future work. The experimental results show that film breakup occurs already at $x / c \approx 20 \%$. This corresponds very well to

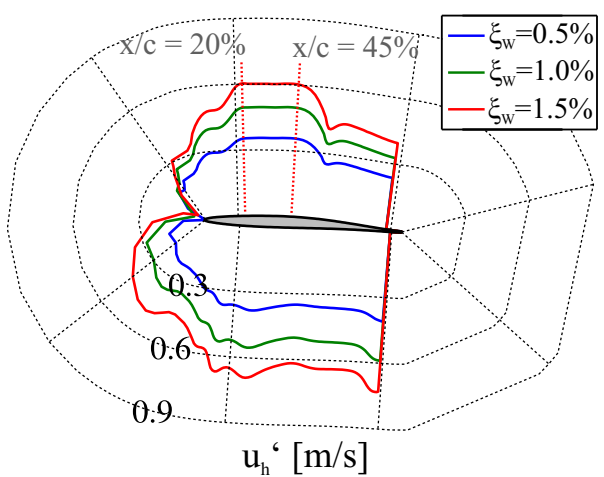

Figure 13. Perturbation velocity $u_{h}^{\prime}$

the area marked with the two red dashed lines in fig. 13. The slope of the curves show a small decrease of $u_{h}^{\prime}$ at the position of $x / c \approx 20 \%$. Taking into account the very small film height $h$ displayed in fig. 12 (a) a slight decrease of the damping of a perturbation seems to be sufficient to cause film breakup. If the film breaks up in one position the fluid is displaced sidewards leading to a slight increase of film thickness next to the position of breakup and the formation of a gas-liquid solid interface. In case of the lowest injection rate the film is very thin $(h \approx 1.8 \mu \mathrm{m})$ and the slight increase film height does not seem to be large enough to give enough volume to withstand the growing perturbations for a long distance. This leads to a very sharp breakup line as shown in fig 10 (a). If the film height is slightly larger, however, the effect of displacement seems to be large enough to lead to a delayed breakup. Keeping in mind the almost horizontal slope in fig 13 the perturbations are neither dampened nor instigated to a great extent in the area of $x / c=20-45 \%$. This explains the experimental findings for the larger water loads showing a longer region of film breakup. Regarding the film on the pressure side no film breakup was seen in the experiments. Although a decreasing value of $u_{h}^{\prime}$ is present on the pressure side, as seen in fig. 13, also indicates film breakup the larger film source term and the consequently almost constantly growing film height $h$ counteract this effect. It is seen that the slope of $u_{h}^{\prime}$ provides an indication of the position of film breakup but additional information is needed to precisely determine a breakup position.

In summary the experimental and numerical results are in good agreement.

\section{CONCLUSION}

This paper presents experimental and numerical results for the breakup of a thin liquid film which is formed on stationary compressor blades under overspray fogging conditions. The experiments were conducted at the transonic two-phase wind tunnel at Helmut-Schmidt University in Hamburg, Germany while the numerical investigations were carried out in the Institute of Aerospace Thermodynamics at Stuttgart University, Germany. An experimental setup was introduced and an image processing algorithm was presented to analyze the images of 
the water film pattern on the suction side of the blade. The algorithm was validated using a calibration plate with two different backgrounds. The validation showed a sufficiently high accuracy to determine the position of rivulets corresponding to the position of film breakup. The experimental results of the test case revealed that the onset of film breakup is almost independent of the water load but the area is increased with a higher water load.

A numerical model for the determination of film breakup was presented. The prediction is based on classical linear stability analysis and the consequent analysis of the damping and exciting forces influencing the perturbation. Film breakup is indicated to be at the position in which the acting forces lead to a deceleration and a consequent excitement of the perturbations. The model is applied to a test case and the results show good agreement with the experiments in terms of the onset position of film breakup and the tendency for an increased a water injection.

Thus, with this paper a first insight on film breakup is given for overspray fogging relevant boundary conditions showing the benefits of detailed experimental and numerical work.

\section{ACKNOWLEDGMENTS}

This work has been partly funded by the German Science Foundation (Deutsche Forschungsgemeinschaft). The authors kindly acknowledge the financial support for this work by the German Science Foundation.

\section{NOMENCLATURE}

$c$

$D_{10}$

$D_{32}$

h

$\dot{h}$

$M a_{\text {design } / \text { in }}$

$p_{G}$

$t_{\text {shutter }}$

$T_{\text {tot,in }}$

$\overline{\mathbf{u}}$

$u_{h}^{\prime}$

$w$

$x$

$\beta_{\text {in } / \text { out }}$

$\Delta \beta_{\text {design }}$

$\Phi$

$\kappa$

$\mu_{L / G}$

$\rho_{L / G}$

$\sigma_{\text {gauss }}$

$\sigma$

$\tau_{c}$

$\tau_{G}$

$\tau_{I}$

$\xi_{w}$ chord length

Mean diameter

Sauter mean diameter

wall film height

net mass source term

Design/inlet Mach number

gas pressure

shutter time

Total temperature at inlet of the test rig height average film velocity

perturbation vleocity

air velocity

flow direction

inlet/outlet flow angle

design deflection angle of the cascade

potential

substrate curvature

Liquid/gas dynamic viscosity

Liquid/gas density

standard deviation for Gaussian filter

surface tension

Characteristic time $\tau_{c}=\left(\rho_{L} d_{L}^{2}\right) /\left(18 \mu_{G}\right)$

Wall shear due to co-flowing gas

Wall shear due to impacting droplets

Water mass fraction

\section{REFERENCES}

[1] N. Krzikalla, S. Achner, and S. Brühl. Possibile solutions for compensation of fluctuating energy supply by renewable energies. Study commissioned by German federal Organization for Renewable Energies (in German), pages 16.1-16.13, 2013.

[2] R.K Bhargava, C.B. Meher-Homji, M.A. Chaker, M. Bianchi, F. Melino, A. Peretto, and S. Ingistov. Gas Turbine Fogging Technology: A State-of-the-Art Review: Part I-Part III. Journal of Engineering for Gas Turbines and Power, 129:443-472, 2007.

[3] L. Balling. Fast cycling and rapid start-up: New generation of plants achieves impressive results. Modern Power Systems, 1, 2011.

[4] C. B. Chaker, M.A. andMeher-Homji. Effect of Water Temperature on the Performance of Gas Turbine Inlet Air-Fogging Systems. ASME Turbo Expo, GT2013-95956, 2013.

[5] B.E. Thompson and Juneho Jang. Aerodynamic Efficiency of Wings in Rain. Journal of Aircraft, 33(6):1047-1053, 1996.

[6] F.G. Hammitt, S. Krzeczkowski, and J. Krzyzanowski. Liquid film and droplet stability consideration as applied to wet steam flow. Forsch. Ing.-Wes., 47(1):1-36, 1981.

[7] H. Gomaa and B. Weigand. Modelling and investigation of the interaction between drops and blades in compressor cascades with a droplet laden inflow. ISROMAC-14, 2012, 2012.

[8] B. Ober. Experimental Investigation on the Aerodynamic Performance of a Compressor Cascade in Droplet Laden Flow. Helmut-Schmidt University, Hamburg, Germany, 2013.

[9] A.H. Lefebvre. Atomisation and Sprays. Combustion. Taylor \& Francis, 1. edition, 1989.

[10] M. Chaker and C.B. Meher-Homji. Gas Turbine Power Augmentation: Parametric Study Relating to Fog Droplet Size and its Influence on Evaporative Efficiency. ASME Turbo Expo, GT2008-51476, 2008.

[11] H.-E Albrecht, M. Borys, N. Damaschke, and C. Tropea. Laser Doppler and Phase Doppler Measurement Techniques. Springer, Berlin and New York, 2003.

[12] K. Zuiderveld. Graphics Gems IV: Chapter: Contrast Limited Adaptive Histogram Equalization. Academic Press Professional, Inc., San Diego, CA, USA, 1994.

[13] J Canny. A Computational Approach to Edge Detection. IEEE Trans. Pattern Anal. Mach. Intell, 8(6):679-698, 1986.

[14] R. Kapulla, J. Tuchtenhagen, A. Müller, K. Dullenkopf, and H.-J. Bauer. Droplet Sizing Performance of Different Shadow Sizing Codes. Lasermethoden in der Strömungsmechanik, 16. Fachtagung, Deutsche Gesellschaft für Laser-Anemometrie GALA e.V., 2008. 
[15] H. Gomaa. Modeling of Liquid Dynamics in Spray Laden Compressor Flows. PhD Thesis at the University of Stuttgart, Germany, 2014.

[16] ANSYS INC COOPERATION. ANSYS CFX-Solver Theory Guide. Release 14.0, 2011.

[17] J.R. Dormand and P.J. Prince. A family of embedded Runge-Kutta formulae. Journal of Computational and Applied Mathematics, 6(1):19-26, 1980.

[18] P.J. O'Rouke and A.A. Amsden. The tab method for numerical calculation of spray droplet breakup. SAE Paper, (872089), 1987.

[19] J.K. Dukowicz. A particle-fluid numerical model for liquid spray. Journal of Computational Physics, 35(2):229-253, 1980. 